\title{
BMJ Quality Improving Outpatient Clinic Experience for Core Medical Trainees
}

\author{
Natalie King, Catherine Zhu
}

To cite: King N, Zhu C. Improving Outpatient Clinic Experience for Core Medical Trainees. BMJ Quality Improvement Reports 2017;6:u221836.w8226 doi:10.1136/bmjquality. u221836.w8226

Received 12 December 2016 Revised 21 February 2017

Royal Free Hospital

Correspondence to Natalie King natalie.king14@nhs.net

\section{ABSTRACT}

Outpatient clinic experience is an important component of core medical training. Trainees are expected to attend up to 40 clinics, with a minimum requirement of 24, over the two-year programme. ${ }^{1}$ Yet on a local and national level they have reported difficulties with attending even the minimum number of clinics, largely due to ward commitments and service demands. ${ }^{5}$ A survey of local core medical trainees revealed a baseline mean clinic attendance of 0.5 clinics per month, with only $13 \%$ of trainees having attended the minimum number of clinics. The project aimed to increase the mean clinic attendance to one clinic per month, which would enable trainees to meet their curriculum requirements.

Clinic attendance data was collected from core medical trainees at two-monthly intervals, to coincide with rotation changeover. The problem was initially discussed at our local medical faculty meeting and interventions were proposed. Firstly, an up to date clinic timetable was distributed and consultants encouraged to invite their trainees to clinic. Subsequently a clinic booking system was implemented, to enable trainees to arrange protected time in which to attend outpatient clinics. This intervention was unsuccessful in improving clinic attendance. A revised system of pre-allocating protected clinic time was therefore devised and implemented, which resulted in an increase in clinic attendance figures to above the target. Trainees have been allocated clinic days for the rest of the year, which should enable them to meet their curriculum requirements.

Through the use of PDSA cycles, we were able to rapidly determine the effect of our interventions and make improvements that have led to an increase in trainee clinic attendance. This is a sustainable model that could be easily implemented by other hospital trusts for core medical trainees.

\section{PROBLEM}

By the end of their two-year training programme, core medical trainees in the United Kingdom are required to have gained experience in 24 outpatient clinics. This requirement is checked at the 'Annual Review of Competence Progression' (ARCP), a formal meeting where each trainee's progress through the training programme is reviewed.
Trainees must have satisfactorily achieved the competences outlined in the core medical training (CMT) curriculum before they may progress to higher specialty training. The Joint Royal College of Physicians Training Board (JRCPTB) Quality Criteria 2015 states that trainees should ideally attend a minimum of 40 clinics during this period. ${ }^{1}$ Managing to attend outpatient clinics is one of the aspects of CMT that our local trainees find most challenging. Many struggle to attend even the minimum number of 24 clinics. This not only puts them at risk of failing to achieve completion of training, but also denies them of valuable specialty experience to be gained from participating regularly in outpatient clinics. This is recognised as important preparation for progression to specialty training, where they will be expected to manage outpatients with acute and chronic conditions. ${ }^{2}$

Our trust, a district general hospital, trains fourteen full time core medical trainees. This project was motivated by the difficulties that trainees were having in attending even the minimum number of 24 clinics. Cardiology was the only medical specialty that provided trainees with protected time to attend clinics. Unfortunately, not all trainees had the opportunity to rotate through Cardiology, and some of those that did were marked down at their ARCP for not having attended a variety of clinics. In the other medical specialities, trainees were not allocated any protected time to meet their clinic requirements, which meant they had to independently find time to leave their ward and seek an appropriate clinic to attend. Following informal discussion with current trainees, it became clear that with staff shortages, bed pressures and increasing numbers of medical outliers, this was a challenging task. Some trainees had come in on their days off in order to catch up with their clinic attendance, which was clearly unacceptable.

The project aimed to firstly understand the barriers that core medical trainees faced in 
attending outpatient clinics. To meet the minimum requirements, trainees would need to attend, on average, one clinic per month. As the problem with attendance appeared to lie with those specialities that did not offer protected clinic time, Cardiology clinics were not included in the data collection, to prevent introducing bias into the results. We aimed to introduce methods to improve clinic attendance and enable trainees to attend at least one clinic per month, without having to attend in their own time. The time frame set for achieving this was nine months.

\section{BACKGROUND}

CMT is a two-year training programme in general medicine that bridges the gap between the Foundation programme (two years of supervised clinical practice following qualification) and Specialty training. Its aims are to prepare trainees for taking on a senior role, where they will be required to lead the acute medical take and manage patients with acute and chronic conditions in both an inpatient and outpatient setting. ${ }^{2}$ The curriculum requires trainees to have achieved satisfactory performance in 24 outpatient clinics by completion of CMT. Yet, in line with the JRCPTB Quality Criteria, trainees are advised to aim to attend 40 clinics over the two years, where there is the facility to do so. ${ }^{3}$ The Quality Criteria suggests that bleep-free cover arrangements should be in place to allow trainees to attend outpatient clinics. ${ }^{1}$

The CMT curriculum defines a medical clinic as an activity involving care of patients in a scheduled manner, including patients newly referred and those returning for follow up. The trainee should have the opportunity to review patients in clinic under supervision, interpret and order appropriate investigations, and explain the results or management plan to the patient. ${ }^{4}$ They are required to document their participation in clinics using the ePortfolio, an electronic tool for doctors to record evidence of their competencies, and obtain feedback through use of workplace-based assessments.

A survey of UK core medical trainees in 2013 showed that difficulty in attending outpatient clinics is a national problem. Most trainees reported that service provision took up most of their time at work, with significant loss of training opportunities. Over one third of trainees attended ten or fewer outpatient clinics per year. $81 \%$ of trainees were frequently prevented from attending clinics due to inpatient commitments. $71 \%$ felt they should go to more outpatient clinics than they were currently attending, with most trainees evaluating clinic experience as 'valuable' or 'very valuable' to their training. ${ }^{5}$

\section{BASELINE MEASUREMENT}

Initial data collection aimed to obtain both quantitative and qualitative data of the existing problem. A survey was distributed to trainees and completed anonymously.
Trainees were asked how many clinics they had attended in the previous six months and in the two-month clinical rotation they had just completed. They were asked explain the factors that prevented them from attending more outpatient clinics and suggestions as to how clinic attendance could be improved.

Eight responses were collected from the initial survey. Only one trainee $(13 \%)$ had attended the minimum target of six clinics in the preceding six months (a mean of one clinic per month). The overall mean clinic attendance was 0.5 clinics per month, with two trainees $(25 \%)$ not having attended any clinics in their first six months of core medical training. These results indicated that most trainees were not on track to achieving the minimum number of clinics required by the end of the training programme. In the preceding two-month rotation, the mean clinic attendance was just 0.38 and, again, only one trainee $(13 \%)$ had met the target of one clinic per month.

Survey responses highlighted ward commitments as the main reason preventing trainees from attending clinics. Most trainees reported they were too busy with service provision, to be able to safely leave the ward. Staffing levels were highlighted as an important barrier, as well as core medical trainees feeling that they could not burden their more junior colleagues to take on the entire workload of the ward. If registrars were off the ward, the core medical trainee would be the most senior doctor present. In the event that they did make it to clinic, they were likely to be bleeped, resulting in distractions or even having to leave the clinic to attend to a ward problem. Trainees also felt there was a low expectation, with little encouragement, from their consultants for them to attend their specialty clinics.

Based on this initial data, we decided to collect clinic attendance figures at the end of each two-month rotation. Trainees would simply be asked at the end of each two-month rotation how many clinics they had attended. From this we would calculate two outcome measures: the mean clinic attendance per month and the percentage of trainees that had met the target of one clinic per month. This would give an overview of how many clinics trainees were attending in each particular specialty rotation and allow time to introduce and see the effects of interventions.

\section{DESIGN}

1. The first step was to discuss the problem at the local medical faculty meeting and propose interventions. This was attended by the medical consultants, including our CMT college tutor, who oversees the local training programme. The initial data, showing the poor clinic attendance record, was presented and the reasons why trainees were unable to attend clinics discussed. It was felt that, while desirable, it would not be possible to rota CMTs regularly into clinics in every medical specialty, in the same way that is done 
in Cardiology. This was due to a lack of junior medical staff to cover the wards and also because of a lack of space available in outpatients. In order to ensure that CMTs would be able to attend one clinic per month, we proposed introducing protected clinic time that could be booked by the CMTs in advance, thus ensuring that there would be enough juniors present to cover the wards. Each CMT would be entitled to book one day per two-month rotation in which to attend clinics. The rota coordinator would ensure that staffing levels were safe on the ward and book the clinic time onto the rota. The meeting also served to raise awareness of the training problem among the medical consultants. This was partly in response to the trainee feedback that there was a low expectation from their consultants for them to join them in clinic. Clinical supervisors were asked to encourage their core medical trainees to attend clinic, particularly if at the morning board round there appeared to be enough other junior doctors present to cover the ward.

2. The next intervention introduced was to create an up to date clinic timetable of which specialty clinics were taking place on each day and with which consultant. This was to equip trainees with the knowledge of which clinics were available for attendance if ward commitments allowed. Trainees were also encouraged to use this timetable to try to pre-arrange attending clinics with their clinical supervisors. The clinic timetable was emailed out to all trainees.

3. Once the medical consultants and rota coordinator had been informed of the new protected clinic booking system, trainees were instructed how to utilise it and the system was rolled out. Trainees were informed by email and also in person at the weekly lunchtime teaching session.

\section{STRATEGY}

PDSA cycle 1

Following the initial data collection, we discussed the results at a CMT lunchtime teaching session where the majority of trainees were present. The problem was discussed further, with ideas proposed for addressing the problem. Most trainees felt that implementation of protected, bleep-free time would be most effective in enabling them to attend clinics. If they were allocated protected time, this would help reduce ward commitments preventing them from attending. As the response rate to our initial questionnaires was only 8 out of 14 , trainees were encouraged to participate in future data collection for the project, as it was in their training interests.

The problem, with proposed interventions, was then discussed at the local medical faculty meeting. A clinic booking system was proposed and discussed, where trainees would be entitled to arrange protected time in which to attend one day of clinics per two-month rotation. This would ensure that all trainees could, in theory, meet the minimum requirements of one clinic per month. Consultants were asked to invite and encourage their core medical trainees to join them in clinics.

A clinic timetable detailing where and when the clinics for all the medical specialities were taking place was put together and sent out to all the trainees. This would help trainees decide which clinics they would like to attend. The clinic booking system took time to set up, which meant that it had not been implemented in time for the next data collection.

After two months (at the end of the trainees' next clinical rotation) trainees were asked how many clinics they had attended. The response rate was better with eleven trainees replying. Mean clinic attendance had risen slightly to 0.55 clinics per month, but was still far short of our target. The percentage of trainees achieving one clinic per month increased to $27 \%$, however six trainees $(55 \%)$ had not attended any clinics at all. Two trainees managed to attend four clinics, which was the highest number achieved in this time period.

\section{PDSA Cycle 2}

The clinic booking system was rolled out once preparations were in place. The rota coordinator was informed and willing to participate with the proposed system. Trainees were instructed to book clinic time with the rota coordinator, ensuring that there were enough trainees remaining on the wards. This would be documented on the rota, in the same way as annual or study leave. Trainees could book one day to use to attend clinics per two-month rotation. They were advised to use the clinic timetable to plan ahead which clinics they would like to attend and ideally to inform the relevant consultant beforehand.

At the next data collection, after having rolled out the booking system, the mean clinic attendance unfortunately decreased. Of the eleven trainees that responded, two had attended one clinic in the two months. The rest had attended zero clinics. The mean clinic attendance dropped to 0.09 clinics per month and $0 \%$ of trainees achieved the minimum target.

\section{PDSA Cycle 3}

Following the introduction of the clinic booking system, the results of the project so far were presented to the trainees and the CMT college tutor. Feedback on the clinic booking system was obtained from the trainees. Unfortunately its uptake had been poor for several reasons. Many had not managed to find time to organise their clinics. Others had found that they were unable to book in clinics, as there were already too many trainees away from the ward due to annual or study leave. Trainees reported that they would prefer for clinic days to be pre-allocated to them on the rota well in advance. If a trainee wanted to attend a particular clinic, they could always arrange to move their clinic day. 
Following this discussion, it was agreed with our CMT college tutor that we would introduce for clinic days to be put on the rota in advance by the rota coordinator. One clinic day per two-month rotation would be allocated to each trainee. This was put on the rota prior to its release, which meant that it was not affected by other trainees' leave arrangements. This was put in place ready for the changeover of trainees in August and clinic days were allocated for the entire year ahead.

The introduction of allocated protected time led to a vast improvement in clinic attendance and the minimum mean attendance was achieved. At the next data collection, thirteen trainees responded to the survey. Ten trainees $(77 \%)$ had achieved at least a mean of one clinic per month. Five trainees had managed to go to further clinics outside of their protected days. The mean clinic attendance had risen dramatically to 1.15 clinics per month. Yet there were still three trainees who did not attend any clinics in the two months.

\section{RESULTS}

Our outcome measures were mean monthly clinic attendance and percentage of trainees attending one clinic per month. This was collected at two monthly intervals, to coincide with clinical rotation changeover. The exception was where this coincided with trainee changeover in August, at which point we skipped a data collection point. Collecting data at this point would likely have been biased by trainees in their second year already having received their training outcomes.

Our project target was for trainees to attend at least one clinic per month. The initial data collection showed that the baseline mean clinic attendance was below this at just 0.38 clinics per month (figure 1). Only $13 \%$ of trainees were achieving target clinic attendance. Following the medical faculty meeting and the release of an up to date clinic timetable we saw a small increase in attendance, which was still far below our target, at 0.55 clinics. There was also a modest rise in trainees attending one clinic per month, to $25 \%$.

The main intervention, our clinic booking system, was unfortunately unsuccessful and clinic attendance was seen to actually decrease following its introduction $(0.09$ clinics per month). Following feedback from trainees, it appeared that the main reason for this was that trainees did not use it. In theory it had the potential to help them book protected time, but some trainees did not find time to arrange it, while others found there were too many other junior doctors already on annual or study leave at the times they tried to book it. Trainees voiced that they would prefer to be pre-allocated protected time for clinics and we sought to put this into action.

In time for the August changeover of new trainees we arranged protected clinic days for every two-month rotation. The rota coordinator was asked to allocate clinic days to trainees for the whole year. This has proved a success, with the mean monthly clinic attendance having increased to 1.15 , above our target. $77 \%$ of trainees achieved the target clinic attendance, a dramatic rise. Most trainees were able to attend their clinic days without any problem. Only one trainee was unable to attend their clinic day due to their ward being too busy. Two lost their clinic day due to personal swaps of shifts, which trainees have since been discouraged from doing. Consultants have been informed that protected clinic days must be adhered to, except in exceptional circumstances.

\section{LESSONS AND LIMITATIONS}

Lessons learnt from our project were the importance of PDSA cycles when introducing and evaluating interventions. While the introduction of the initial clinic booking system was unsuccessful in improving clinic attendance, establishing this promptly enabled us to obtain feedback as to why it didn't work and what steps we could take to change it. The protected clinic days
Figure 1 Run Chart of Monthly Clinic Attendance

\section{Run Chart of Monthly Clinic Attendance}

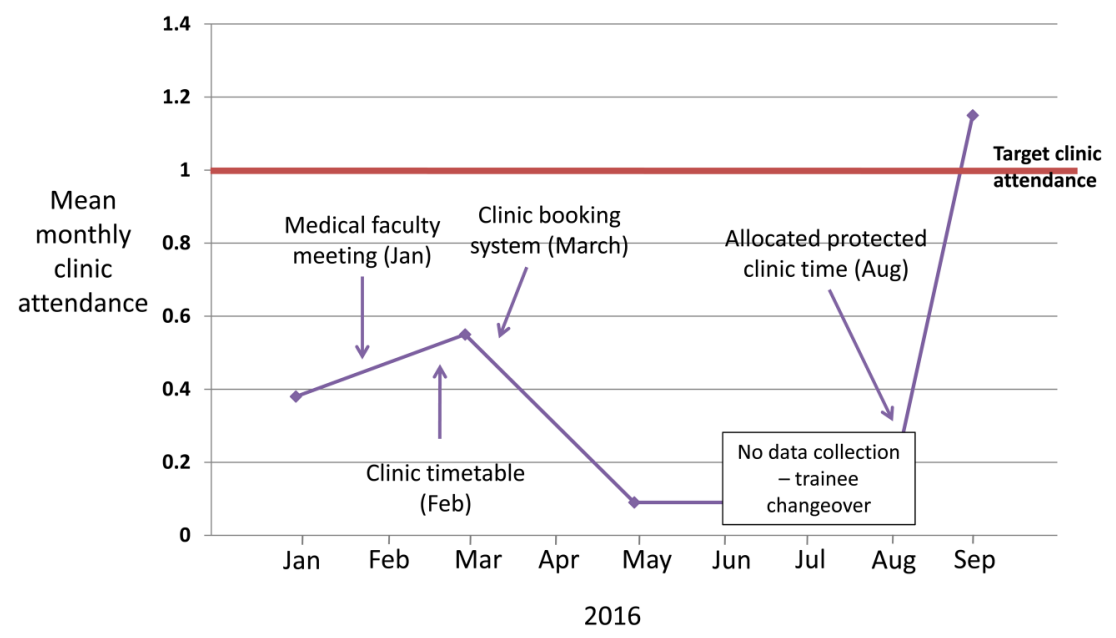


that have been introduced, not only resulted in a dramatic improvement in clinic attendance, but are also a sustainable intervention. The clinic days have been put on the rota for the whole year, which should ensure that trainees can meet their curriculum requirements. It will also give them the important experience in managing patients in an outpatient setting. A current trainee at the hospital is continuing the quality improvement project, so that its longer-term sustainability and success can be determined. In order to ensure that protected clinic time remains on the rota for future cohorts of trainees, we have gained the support from our CMT college tutor and the medical rota coordinator.

One limitation in measuring the mean clinic attendance per month, was that it was subject to bias from extremes of attendance. Trainees who were attending more than the minimum number of clinics per month, may compensate for those who were still not managing to attend, thus giving a false picture of the situation. It was for this reason that we excluded cardiology clinics from the data collection, as this would have falsely skewed the data. The percentage of trainees achieving the target number of clinics dramatically rose to $77 \%$, but this is still off target. $100 \%$ of trainees need to attend the minimum number of clinics in order to pass their ARCP and progress to the next level of training. It is important that this project is continued to ensure the sustainability of the intervention.

A second problem that we faced, which resulted in a further limitation to the project, was not obtaining a full set of responses from trainees. This was worse at the outset of the project, where only 8 responses were received out of a total of 14 trainees. This limited the accuracy of our initial data. After meeting with trainees to make them more aware of the purpose of the project and the proposed changes, they perhaps realised that it was in their interests to respond to the questionnaires. As the project progressed, the response rates increased and trainees more readily participated in data collection. The subsequent response rates were 11 or above at each data collection. Although it would be most ideal to have a full set of responses, these sample sizes give enough data to see the true effects of each intervention.

This project is also limited in that, while it has improved the number of clinics attended by core medical trainees, we cannot guarantee that each trainee will have a satisfactory learning experience during a clinic. Lack of available space in outpatients was one problem raised at the medical faculty meeting. While it is good experience to observe a consultant running a clinic, it is preferable for the trainee to have a room in which to review patients themselves and obtain feedback. Ensuring a high quality clinic experience for the trainee will be the next stage of this project. Trainees should have the opportunity to see patients themselves, order investigations and formulate management plans, with supervision from a consultant. They should also have the chance to complete workplace-based assessments and obtain feedback.

As well as measuring the effectiveness of the intervention, it is also important to consider the balancing measures. When discussing the initial problem of CMT clinic attendance, we highlighted ward commitments and staff shortages as factors that prevented trainees from going to clinics. Our hospital has a specified minimum number of doctors that should be present on each ward, to ensure patient safety. This limits how many doctors can be away from the ward due to annual leave, zero days or study leave at one time. By introducing protected clinic time that is documented on the rota at the start of the year, we aimed to ensure that CMTs would get priority over other types of leave in order to attend clinics. As the intervention continues it will be important to gain feedback on how the intervention affects other types of trainees, such as foundation doctors and GP trainees. Having protected clinic time for CMTs, could make it difficult for others to obtain leave and could result in times when junior colleagues are less supported in covering the ward. It will also be important to determine the views of consultants on whether it has had any impact on how efficiently and safely the ward is run.

\section{CONCLUSION}

A survey of core medical trainees in the United Kingdom in 2013 suggested that the problem of poor outpatient clinic attendance is not unique to our trust, but national. ${ }^{5}$ Trainees were prevented from attending outpatient clinics due to service provision and inpatient commitments, on both a national and local level. We have devised a sustainable system of allocated protected clinic time to improve trainee clinic attendance. This intervention could be easily replicated at other trusts where core medical trainees are not meeting their clinic requirements, with the involvement of their local training programme director and rota coordinator. This intervention does not have to be restricted to core medical training and could be used by other specialties where trainees would benefit from clinic experience but are not currently timetabled to attend.

The use of improvement cycles has enabled us to meet our initial aim of increasing clinic attendance so that trainees would meet their curriculum requirements. The monthly clinic attendance has risen to 1.15 clinics per month since pre-allocated protected time has been introduced. $77 \%$ of trainees used their allocated clinic days in the last two-month rotation. Ensuring that trainees experience sufficient clinic time during CMT will better equip them for taking on specialty registrar training posts and give them experience in managing conditions that they may not see on the wards. Improving the education of our trainees, will lead to better patient care in the present and future. The future of this project will aim to ensure the sustainability of our intervention and improve the quality of clinic experience for trainees. 
Acknowledgements I would like to thank our CMT college tutor, Dr Ramabhadran Balaji, and rota coordinator, Leah Davis, for their support in introducing protected clinic time for trainees.

Declaration of interests Nothing to declare.

Ethical approval Ethical approval was not sought, as this is a quality improvement project that aimed only to improve the attendance at outpatient clinics by core medical trainees. The project did not provide generalizable knowledge in a field of inquiry, therefore ethical approval was not required.

Open Access This is an open-access article distributed under the terms of the Creative Commons Attribution Non-commercial License, which permits use, distribution, and reproduction in any medium, provided the original work is properly cited, the use is non commercial and is otherwise in compliance with the license. See:

- http://creativecommons.org/licenses/by-nc/2.0/

- http://creativecommons.org/licenses/by-nc/2.0/legalcode

\section{REFERENCES}

1. Joint Royal College of Physicians Training Board Quality Criteria for Core Medical Training (CMT) 2015. https://www.jrcptb.org.uk/sites/ default/file/0711_JRCPTB_CMT_A4_4pp_WEB.pdf (accessed 17/ $11 / 16)$

2. Joint Royal College of Physicians Training Board website. Core medical training and acute care common stem (medicine) https:// www.jrcptb.org.uk/specialties/core-medical-training-and-acute-carecommon-stem-medicine (accessed 20/11/16)

3. Core Medical Training Decision Aid - Revised September 2015 https://www.jrcptb.org.uk/sites/default/files/CMT\%20ARCP\% 20Decision\%20Aid\%20\%28September\%202015\%29.pdf (accessed 20/11/2016)

4. Specialty Training Curriculum for Core Medical Training August 2009 (Amendments 2013) https://www.jrcptb.org.uk/sites/default/files/ 2009\%20CMT\%20Curriculum\%20\%28amendments\%202013\%29\% 20100516.pdf (accessed 20/11/16)

5. Tasker F, Newbery N, Burr B, Goddard AF. Survey of core medical trainees in the United Kingdom 2013 - inconsistencies in training experience and competing with service demands. Clinical Medicine (London) 2014;14:149-56 